\title{
Magnetic field components analysis of the SCUPOL 850 microns polarization data catalog
}

\author{
Frédérick Poidevin, Diego Falceta-Gonçalves, Grzegorz Kowal, \\ Elisabete De Gouveia Dal Pino and Antonio-Mário Magalhães
}

University College London, Kathleen Lonsdale Building, Department of Physics \& Astronomy, Gower Place, London WC1E 6BT, United Kingdom

email: Poidevin@star.ucl.ac.uk

\begin{abstract}
The SCUPOL catalog is a compilation of 83 regions that were observed at the JCMT between 1997 and 2005. For sufficiently sampled maps, we conduct an analysis for characterizing the polarization and magneto-turbulent properties of the observed regions. The same analysis is done on 2D polarization maps produced by 3D MHD 1024 pixel grid simulations that have been scaled on a sample of observed maps. Each scaled MHD cube of simulated data is used to calculate the mean turbulent regime of each observed regions.
\end{abstract}

Keywords. ISM: magnetic fields, ISM: molecular clouds, polarimetry, submm, MHD: simulations

We present an analysis of the SCUPOL catalog produced by Matthews et al. (2009).

For sufficiently sampled maps of star-forming regions, inferred parameters $(<\mathrm{p}\rangle, \gamma, \mathrm{b})$ are estimated to characterize the polarization properties, the depolarization properties and the turbulent-to-mean magnetic field ratio of each region as seen on the plane-ofsky. Statistical studies show no specific correlation of each parameters with each other suggesting that they provide information of different nature about the region considered, at least at the $14^{\prime \prime}$ resolution of the observations. Similar set of parameters are calculated from 2D polarization maps produced with 3D MHD 1024 pixel grid simulations (see Falceta-Gonçalves et al. (2008)) performed for different MHD regimes. Such MHD regimes have been estimated for 4 regions with 3D MHD cubes properly scaled on the observed maps. Our results shown in Tab. 1 and those obtained by Crutcher (1999)'s analysis of Zeeman Measurements toward subregions of the clouds are consistent within a factor 2 .

Table 1. Description of the simulations - MHD, $1024^{3}$.

\begin{tabular}{|l|c|c|c|}
\hline Model & $\mathbf{M}_{S}^{(a)}$ & $\mathbf{M}_{A}^{(b)}$ & Object Name \\
\hline $1 \ldots \ldots \ldots$ & 2.0 & 0.3 & $\mathrm{~S} 106$ \\
$2 \ldots \ldots \ldots$ & 3.0 & 0.5 & $\mathrm{OMC}-2 / 3$ \\
$3 \ldots \ldots \ldots$ & 5.0 & 0.7 & $\mathrm{~W} 49$ \\
$4 \ldots \ldots \ldots$ & 5.0 & 0.7 & $\mathrm{DR} 21$ \\
\hline
\end{tabular}

Notes: ${ }^{(a)}$ Sonic Mach Number. ${ }^{(b)}$ Alfvénic Mach Number.

\section{References}

Crutcher, R. 1999, ApJ, 520, 706

Falceta-Gonçalves, D., Lazarian, A., \& Kowal, G. 2008, ApJ, 679, 537-551

Matthews, B. C., McPhee, C., Fissel, L., \& Curran, R. L. 2009, ApJSS, 182, 143 